\title{
Utilizing chick embryo in ovo model system to study the effects of misoprostol on early embryogenesis - a pilot study
}

\begin{abstract}
Misoprostol, a synthetic prostaglandin Elanalogue, is an effective anti-ulcer drug as well as prescription drug for abortion induction. It is also illegally used for unsupervised self-induction of pregnancy termination. However failed abortion, leads to embryo exposure to the drug and is reported to result in congenital anomalies.

The current study reports preliminary results of a pilot project designed for evaluating the effects of misoprostol administration on developing chick embryos. The project comprised of two groups of fertilized chicken eggs. The groups were labelled as "A" for control $(\mathrm{n}=5)$ and "B" for treated $(\mathrm{n}=5)$ specimens. The treated group embryos were exposed to $4 \mu \mathrm{g}$ of misoprostol by injecting the drug directly into the yolk sac, while same volume of normal saline was injected in the control group. Whole mount examination of sacrificed embryos after 33 hours of incubation revealed normal stage of development (HH- stage 10) in the control group embryos. While in the treated group, 02 out of 05 embryos lagged in their developmental stage (HH- stage 8), with no brain vesicles formation and lesser number of somite pairs.
\end{abstract}

It is concluded that exposure of developing chick embryos to Misoprostol in a corresponding therapeutic dose of adult females can potentially cause developmental delay and growth regression of the embryos.

Keywords: chick embryos, misoprostol, congenital anomalies, somites, neural tube
Volume 2 Issue I - 2017

\author{
Nusrat Zareen,' Nosheen Karim, ${ }^{2}$ Lubna \\ Akhtar, $^{3}$ Jaweria Faisal ${ }^{4}$ \\ 'Department of Anatomy, Isra University, Pakistan \\ ${ }^{2}$ Department of Immunology, Northern Borders University, KSA \\ ${ }^{3}$ Department of Anatomy, Foundation University, Pakistan \\ ${ }^{4}$ Department of Gynecology \& Obstetrics, Isra University, \\ Pakistan
}

Correspondence: Nusrat Zareen, Department of Anatomy, Isra University, Lehtrar Road, Farash Town, Phase II, Islamabad, Pakistan, Tel (+92 5I) 843990I I0, Fax (+92 5I) 8439900, Email nusrat.zareen@gmail.com

Received: December 05, 2016 | Published: January 24, 2017

\section{Introduction}

Misoprostol is a synthetic prostaglandin $E_{1}$ analogue used for a variety of indications in medical practice including medication of abortion. ${ }^{1}$ In addition to therapeutic usage, an unsupervised and illegal self-induction trend of Misoprostol administration has promoted its use as an illegal abortifacient. ${ }^{2}$ It is reported that $15 \%$ of misoprostol-induced-abortions may not be successful, even under medical supervision. This leads to in utero exposure of the drug to the developing fetus. ${ }^{3}$ It is scientifically claimed that use of misoprostol with failure to abort may be linked to birth defects suggesting an $8-10 \%$ risk of abnormalities among women who use misoprostol and experience abortion failure. ${ }^{4}$

The etiology of congenital malformations caused by misoprostol is thought to be due to a vascular disruption secondary to uterine contractions caused by the drug. ${ }^{5}$ Neonates exposed to Misoprostol in utero are reported to present with various congenital defects including skull defects, bladder exstrophy, arthrogryposis, cranial nerve palsies, facial malformations, terminal transverse limb defects, and Moebius sequence. ${ }^{6}$

However most of these deformities are reported in retrograde studies, based on positive history of mothers for misoprostol induction. ${ }^{7}$ The sparse data of focused experimental studies, with live embryo exposure and control comparison, leaves gaps in facts for establishing the teratogenic effects of misoprostol and other drugs. ${ }^{8}$ Keeping in view these gaps in knowledge, an animal based experimental study is designed, selecting chick embryos as study subjects, to study the dose related effects of misoprostol on early embryogenesis. The present study reports the observed results of the pilot project for this future planned study, describing also the dose standardization and drug administration methodology.

\section{Materials and methods}

An experimental research design was piloted at the Postgraduate laboratory of Al Nafees Medical College, Isra University Islamabad. Ten freshly laid fertilized chicken eggs $(\mathrm{N}=10)$, belonging to 'Rhode Island Red' breed of Gallus Domesticus, were collected from Poultry Research Institute, Rawalpindi, using random sampling method.

These fertilized eggs were categorized into 2 groups; Control A (for normal saline administration) and treated B (for misoprostol administration), both comprising of 5 eggs each. After sterilizing the egg shells with $70 \%$ alcohol swabs, they were placed in the egg racks with the blunt end facing upwards and the pointed end downwards for about 15 minutes. This permitted the blastoderm to rotate and position near the blunt end avoiding any possible damage while injecting the fluid at the lower end.

Misoprostol was injected in group B embryos by drilling two holes; first a finger breadth above the lower end for injecting the drug and second at the top of the blunt end to allow escape of air normally present at this end. This method was considered suitable for injecting the drug to prevent the leak back of the drug. 
The dose of misoprostol for induced abortion in first trimester of pregnancy is $800 \mu \mathrm{g}$ stat in an adult of $70 \mathrm{~kg} .{ }^{5}$ The corresponding therapeutic drug dose for chick embryos was calculated. Keeping in the view the average weight of $39 \mathrm{gm}$, the equivalent dose in chicken egg was $4.4 \mu \mathrm{g}$. Fluid drug suspension of Misoprostol was prepared by dissolving $200 \mu \mathrm{g}$ of drug in $2 \mathrm{cc}$ of normal saline (i.e. $1 \mathrm{ml} / \mathrm{cc}$ contained $100 \mu \mathrm{g}$ ). Insulin syringe of $1 \mathrm{ml}$ (with 50 div/units) was used to inject $2 \mathrm{U}$ of fluid suspension, administrating $4 \mu \mathrm{g}$ of drug as whole body exposure.

Similarly, the same volume of normal saline was injected in the eggs of the control group A. Soon after the administration of Misoprostol or normal saline, the holes in the shell were sealed with tape and the eggs were placed in the incubator. The temperature was maintained at $38 \pm 0.5^{\circ} \mathrm{C}$, relative humidity was kept between $60-70 \%$ and adequate ventilation was maintained.

The day when eggs were placed in the incubator was taken as day 0 . The eggs were manually rotated $1 / 2$ turn twice daily and opened manually after 33 hours of incubation. The blastoderms were dissected out (Figure 1A), and whole mounts prepared. The dissected blastoderms were washed in normal saline in a watch glass, by gentle shaking. The washings removed most of the adherent yolk and gave relative clarity to blastoderm. It was then transferred to the glass slide and stretched out by the help of a blunt pointer. A drop of $70 \%$ ethanol was poured on it so that the plane of the tissue was, parallel with the plane of the slide. The mount was visualized directly under Olympus binocular light microscope to observe and compare the development and differentiation stages in both the groups (Figures 1B \& 1C).

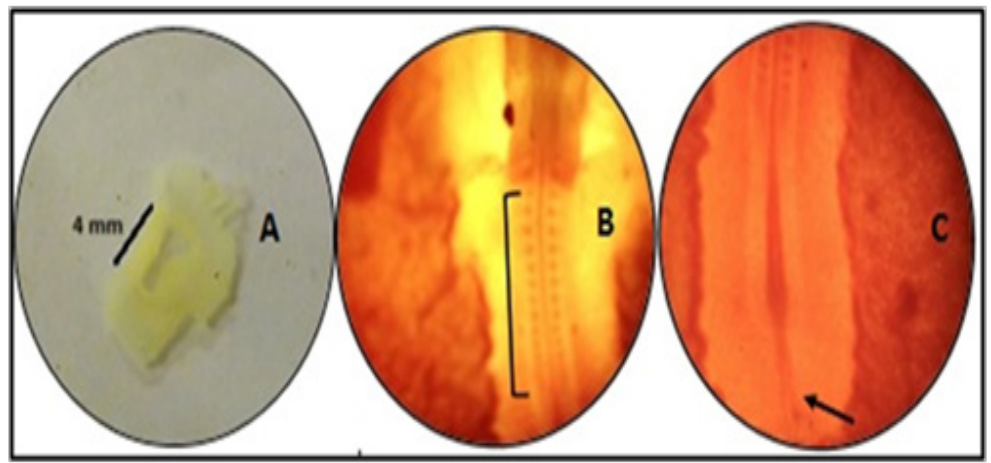

Figure I Whole mount preparation of control embryos.

A: Blastoderm (4mm in length) dissected out.

B: Cranial end of whole mount approximating the HH Stage 10, showing 10 pairs of Somites (in parenthesis)

C: Caudal end of whole mount, showing regressing primitive streak. (Arrow)

\section{Results}

The prepared whole mounts were observed under light microscope at low $(\times 10)$ and high $(\times 40)$ power of magnification. The control chick embryos corresponded to the Hamilton Hamburger HH- Stage 10 of development (33 hours), ${ }^{9,10}$ showing 3 brain vesicles (forebrain, midbrain and hind brain), optic vesicles and $10-11$ pairs of somites, and regressing primitive streak (Figure $2 \mathrm{~A} \& 2 \mathrm{~B}$ ).

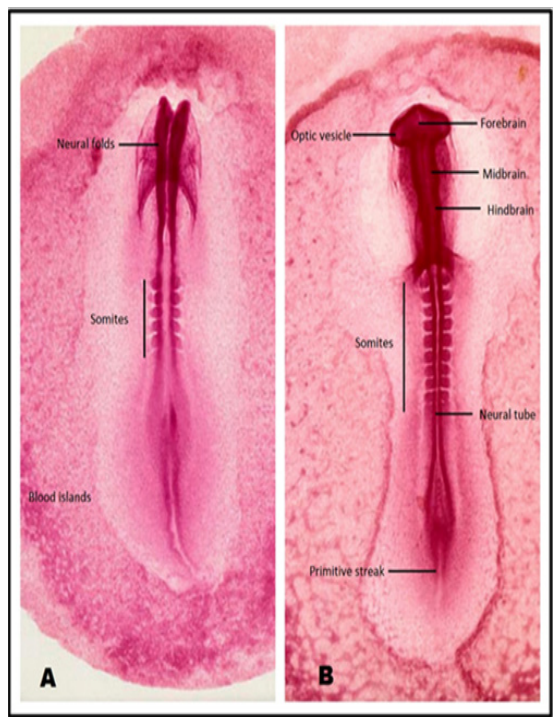

Figure 2 Chick embryo whole mounts - HH staging. ${ }^{30}$

A: Image corresponding to HH Stage 08 (26-19 Hours).

B: Image corresponding to HH Stage 10 (33-39 Hours). 
In the treated group B, 02 out of 05 embryos showed delayed mile stones of development corresponding to Hamilton Hamburger HH Stage 8 of development (26-29 hours). ${ }^{9,10}$ The delayed mile stones and growth retardation was assessed due absent brain dilatations and optic vesicles, and appearance of 4 - 5 pairs of somites only (Figure 2A). The remaining embryos in this group paced with the normal stage of development. However due to lesser number of study and control embryos no statistical comparison was carried out in the current pilot project.

\section{Discussion}

The present study reports a pilot project, designed for evaluating the teratogenic effects of misoprostol, an abortive drug, during early embryogenesis in chick embryos. The project explains the workable methodology of drug dose calculation and administration, along with preliminary parameters of general developmental stages of the embryos.

Misoprostol, although routinely prescribed as an antiulcer drug, is also known for self-prescription and under medical supervision with caution, for inducing abortion. However, if the pregnancy continues, the drug has been reported to cause teratogenicity in the developing fetus. The teratogenic potential of misoprostol is linked to the vascular disruption secondary to uterine contractions. Vascular disruption effects usually occur because of localized or general fetal hypoxia. In humans, fetal defects consistent with vascular disruption pathogenesis have been associated with exposure to cocaine, antihypotensives and abortifacient drugs e.g. Misoprostol. ${ }^{11}$

Vascular disruption is extremely important to developmental process because it can cause dysmorphology at nearly any stage of gestation, and affect almost any tissue or structure. Early histopathological changes in affected tissues reveal that fetal hypoxia leads to edema and an increase in vessel diameter, particularly in distal structures. Loss of fetal blood vessel wall integrity leads to vessel rupture, hemorrhage, and formation of subcutaneous blebs or blood-filled blisters. ${ }^{12}$ Necrosis of affected tissues occurs within 24 to $48 \mathrm{~h}$, and subsequent resorption results in distortion or loss of already formed structures, which may conclude as congenital anomalies. ${ }^{13}$ These anomalies depend on stage of gestation and severity of the insult. Congenital anomalies related to vascular disruption can appear in broad range of development periods including post organogenesis. ${ }^{11}$ Commonly reported vascular disruption defects include adactyly, transverse reduction defects, syndactyly, polydactyly, and club foot. ${ }^{14}$ Most distal parts of the skeleton are the most easily affected by fetal hypoxia; thus, abnormalities of the phalanges as well as facial abnormalities are the most frequent morphologic manifestations of fetal hypoxia. ${ }^{15}$ Other defects often ascribed to vascular disruption include limb body wall complex, gastroschisis, micro-gnathia, anencephaly, limb reduction defects, syndactyly, and orofacial malformations, such as cleft palate. ${ }^{16}$

Research in anatomy, embryology, and developmental biology has largely relied on the use of biological model systems. ${ }^{17}$ Chick embryos make valuable model organism to study vertebrate development. In addition of being cost effective and its easy maintainability, its early embryogenesis involves developmental processes equivalent to those occurring in vertebrates but at a higher pace. ${ }^{18}$ Thirty- three hours' post incubation stage of chick embryos correlate to week 4 of human embryos undergoing the critical period of embryogenesis. Researchers have been utilizing chick embryo model system as a highly effective and manipulative media for recent researches. Yalcin et al. ${ }^{19}$ have utilized ex ovo chick embryo culture system to study the relationships between genetic and microenvironmental factors driving normal and malformed embryonic development. ${ }^{19}$ During the initial days of incubation, a chick embryo, which begins as a blastoderm undergoes gastrulation, neurulation and several morphogenetic movements to form distinct cranial and trunk structures. ${ }^{20}$ These developmental features resemble closely to human development process. Any disruption to these processes can lead to variety of congenital deformities, and the results can be applicable to human embryogenesis too. Utilizing this similarity to human development, scientist have manipulated this effective model system to study the effect various factors including drugs, on chick embryos. ${ }^{21}$ Our study utilizes this model system to study the effects of Misoprostol on early embryogenesis. Use of Misoprostol, an antiulcer drug has been attributed to congenital anomalies. Despite the recommendations to prevent its consumption in pregnancy, especially beyond the gestational ages of viability, the use of misoprostol is reported to be steadily increasing to induce abortion. ${ }^{22}$ As per a cohort study conducted in a Brazilian state, fetuses of pregnant women exposed to misoprostol show a 2.74 times greater risk of congenital anomaly than that of unexposed fetuses. ${ }^{23}$ In 2009, Brasil et al. ${ }^{24}$ also showed a positive association between misoprostol and various types of congenital anomalies but with borderline significance. ${ }^{24}$

Currently the available data for teratogenic effect misoprostol originate mostly from illegal use for abortion and are mainly retrospective, or more recently prospective follow up studies. ${ }^{25}$ This association of the drug and the presenting abnormality in the neonates is dependent on maternal history of drug exposure.

Our project aims at real-life exposure of the developing chick embryos to the drug and actual observation of the embryonic abnormalities appearing in response to it. The findings indicate delayed mile stones relating to the CNS development (absent brain vesicles) and somite appearance (less number of somite pairs) in the embryos. These findings are in consistence with case reports from human data in which growth deficiencies of off sprigs have been reported in failed abortion attempts after administrating misoprostrol. ${ }^{26}$

The neural tube dilatations, predicate the future development of brain \& spinal cord and other related structures e.g. eyes (optic vesicles) \& cranial nerves. Any delay or hindrance to its normal growth of neural tube dilatations can predispose to associated congenital abnormalities. Similarly, abnormal lagging of somites appearance, which normally differentiate into vertebrae, ribs, part of skin, and dorsal wall muscles, limb skeleton and muscle \& bones of the skull can lead to the possibilities of other morphological changes in the differentiation process. Related deformities have been observed by scientists in different human clinical studies. Many organs defects have been reported related to various doses of misoprostol administration after an unsuccessful attempt to induce abortion. Milller et al. ${ }^{27}$ in year 2007, associated ophthalmic manifestation to misoprostol exposure of developing fetuses. In year 2010, a case report claimed to be first ever published case of a human neural defect presenting with holoprosencephly with a history of in utero exposure to misoprostol..$^{28}$ Delayed CNS development, noticed in our study also supports the probability of appearance of neural tube defects in 
misoprostol administered embryos. Also, the observed restriction in the somite number in the study can be linked to the earlier reported musculoskeletal defects due to misoprostol exposure. ${ }^{29}$

Being a pilot project the present report has the limitations of less number of specimen, without statistical comparison of data, fixed dose administration, and no variability of age (stage) of the developing embryos.

\section{Conclusion}

Considering the developmental lag and restriction observed in the treated group, it is concluded that misoprostol can potentially cause growth delay and implicate teratogenic effect on developing chick embryos. The project specifically points to the probable neural tube and musculoskeletal deformities.

\section{Future recommendations}

A detailed study is planned based on the results of this pilot study extending the same methodology, including a range of drug dosages, to be administered at different development stages to observe the toxicity misoprostol on embryogenesis. Other parameters such as cellular bioassays may also be incorporated to further elaborate the scope of the study. Future studies are also recommended for actual morphological effects on developing embryos of different other species e.g. mice, rats, guinea pigs etc, regarding the teratogenic tendencies of misoprostol.

\section{Acknowledgements}

None.

\section{Conflict of interest}

The author declares no conflict of interest.

\section{References}

1. Rebecca A, O'Brien BM. Uses of misoprostol in obstetrics and gynecology. Rev Obstet Gynecol. 2009;2(3):159-168.

2. Cavieres MF. Developmental toxicity of misoprostol: an update. Rev Med Chil. 2011;139(4):516-523.

3. Zordo SD. The biomedicalisation of illegal abortion:the double life of misoprostol in Brazil. Hist Cienc Saude Manguinhos. 2016;23(1):19-36.

4. Rocha J. Brazil investigates drug's possible link with birth defects. BMJ. 1994;309(6957):757-758

5. Mariana BSS, Aline AF, Soraya FH, et al. Misoprostol and teratogenesis in neonates. Braz J Pharm Sci. 2009;45(3):417-422.

6. Allen R, O'Brien BM. Uses of misoprostol in obstetrics and gynecology. Rev Obstet Gynecol. 2009;2(3):159-168.

7. Vauzelle C, Beghin D, Cournot MP, et al. Birth defects after exposure to misoprostol in the first trimester of pregnancy: prospective follow-up study. Reprod Toxicol. 2013;36:98-103.

8. Alexander PG, Clark KL, Tuan RS. Prenatal exposure to environmental factors and congenital limb defects. Birth Defects Res C Embryo Today. 2016;108(3):243-273.

9. Hamburger V, Hamilton HL. A series of normal stages in the development of the chick embryo. 1951. Dev Dyn. 1992;195(4):231-272.
10. Zareen N, Shahid U. Reproducing windowing technique for naked eye observation of chick embryo morphogenesis. Ann Pak Inst Med Sci. 2011;7(3):146-149.

11. Ronald DH. Developmental and reproductive toxicology: a practical approach. 3rd ed. USA: CRC Press; 2011. p. 1168.

12. Danielson MK, Danielsson BR, Marchner H, et al. Histopathological and hemodynamic studies supporting hypoxia and vascular disruption as explanation to phenytoin teratogenicity. Teratology. 1992;46(5):485497.

13. Fantel AG, Barber CV, Carda MB, et al. Studies of the role of ischemial reperfusion and superoxide anion radical production in the teratogenicity of cocaine. Teratology. 1992;46(3):293-300.

14. Gingras JL, Weese Mayer DE, Hume RF, et al. Cocaine and development: mechanisms of fetal toxicity and neonatal consequences of prenatal cocaine exposure. Early Hum dev. 1992;31(1):1-24.

15. Gaily E. Distal phalangeal hypoplasia in children with prenatal phenytoin exposure: results of a controlled anthropometric study. Am J Med Genet. 1990;35(4):574-578.

16. Stoler JM, McGuirk CK, Lieberman E, et al. Malformations reported in chorionic villus sampling exposed children: a review and analytic synthesis of the literature. Genet Med. 1999;1(7):315-322.

17. Cloney K, Franz-Odendaal TA. Optimized ex-ovo culturing of chick embryos to advanced stages of development. J Vis Exp. 2015;95:e52129.

18. Vasiev B, Balter A, Chaplain M, et al. Modeling gastrulation in the chick embryo: formation of the primitive streak. PLoS One. 2010;5(5):e10571.

19. Yalcin HC, Shekhar A, Rane AA, et al. An ex-ovo chicken embryo culture system suitable for imaging and microsurgery applications. $J$ Vis Exp. 2010;44:e 2154.

20. Spurlin J, Lwigale P. A technique to increase accessibility to late-stage chick embryos for in ovo manipulations. Dev Dyn. 2013;242(2):148154.

21. Taskapilioglu MO, Billur D, Kizil S, et al. Targeting apoptosis through Foxp1, and N-cadherin with glatiramer acetate in chick embryos during neural tube development. Turk Neurosurg. 2016;26(4):586-594.

22. Escumalha M, Gouveia C, Cunha M, et al. Neonatal morbidity and outcome of live born premature babies after attempted illegal abortion with misoprostol. Pediatr Nurs. 2005;31(3):228-231.

23. Dal Pizzol TSS, Maria TV, Mengue SS. Exposure to misoprostol and hormones during pregnancy and risk of congenital anomalies. Cadernos de Saúde Pública. 2008;24(6):1447-1453.

24. Brasil R, Coêlho HLL, D’Avanzo B, et al. Misoprostol and congenital anomalies. Pharmacoepidemiol Drug Saf. 2000;9(5):401-403.

25. Vauzelle C, Beghin D, Cournot MP, et al. Birth defects after exposure to misoprostol in the first trimester of pregnancy: prospective follow-up study. Reprod Toxicol. 2013;36:98-103.

26. Adam MP, Manning MA, Beck AE, et al. Methotrexate/misoprostol embryopathy: report of four cases resulting from failed medical abortion. Am J Med Genet A. 2003;123A(1):72-78.

27. Miller MT, Ventura L, Strömland K. Thalidomide and misoprostol: ophthalmologic manifestations and associations both expected and unexpected. Birth Defects Res A Clin Mol Teratol. 2009;85(8):667-676.

28. Pirmez R, Freitas ME, Gasparetto EL, et al. Moebius syndrome and holoprosencephaly following exposure to misoprostol. Pediatr Neurol. 2010;43(5):371-373. 
29. Opaleye ES, Coelho HL, Schüler-Faccini L, et al. Evaluation of the teratogenic risks in gestations exposed to misoprostol. Rev Bras Ginecol Obstet. 2010;32(1):19-35.
30. Dwight K. Chicken Life cycle. Reprinted with permission. 1990 